\title{
A Simple Si Pitch-variable Grating With Shape Memory Alloy Actuator
}

\author{
Non-member Yigui Li (Tohoku University) \\ Member Minoru Sasaki (Tohoku University) \\ Member Kazuhiro Hane (Tohoku University)
}

\begin{abstract}
Summary
In this paper, a novel bulk grating in which its pitch can be changed by the shape memory alloy (SMA) actuator has been fabricated using silicon micromachining technology. The pitch-variable grating is specially designed to change the pitch easily with small force. The grating is designed to assure moderate stress by finite element method (FEM). Using the reactive ion etching, the grating has a high aspect ratio more than 10 . In the diffraction experiment, more than $10 \%$ extension ratio has been obtained. The SMA actuator has been installed to the grating. Due to the two-way shape memory effect, the translation mechanism is simple and is easily controlled.
\end{abstract}

Keyword: Silicon micromachines, RIE, SMA actuator, Transmission type bulk grating, FEM

\section{INTRODUCTION}

Gratings are basic indispensable optical devices and can be used to separate incoming ray into components or to filter it by diffraction depending on optical wavelength[1]. Precision gratings can be written by electronic beam, optical interference or mechanically grinding. The pitch of the conventional gratings is always fixed due to fabrication technique. To make the grating pitch variable opens up a new region of optics since the device design will be changed totally and more flexible optical system can be constructed easily. For example, the pitch-variable grating will be very useful in wavelength division multiplexing fiber communication $[2,3]$. Especially, volume gratings with high aspect ratio, such as fiber Bragg gratings, show the high diffraction efficiency and high wavelength selectivity.

MEMS technology is one of the promising ways to generate variable optical components and systems. Researchers at the University of Wisconsin have developed microelectromechanical tunable infrared filter with magnetic actuators for infrared spectral analysis [4]. The tunable infrared filter is a mechanically deformable structure consisting of an array of parallel metal plates joined by spring flexures. The filter and actuator structures are fabricated using the LIGA process and are made of electroplated permalloy. Researchers at Switzerland have developed wavelength-tunable add/drop fiber for optical networks [5]. By using the thermo-optic effect, the device can be tuned over the full free spectral range of $6.4 \mathrm{~nm}$. Although some other optical gratings have been fabricated by the MEMS technology, in most of the proposed gratings, the diffraction efficiency has been changed by modifying the grating profile with actuators [6,7]. Very few gratings in which the grating pitch is variable have been reported

We have developed a simple pitch-variable grating in which a grating with flexible spring is fabricated by the deep reactive etching of Si substrate. In addition, a novel actuator consisting of two-way shape memory alloy (SMA) is installed to change the grating pitch. Due to the two-way shape memory effect, the translation mechanism is simple and is easily controlled [8]. In this paper, the design, fabrication, and extension performance driven by the SMA actuator are described.

\section{GRATING DESIGN}

The fabricated silicon grating consists of two parts: parallel grating part in the center section and spring part connected with the grating line at each side. The schematic diagram of the proposed pitch-variable transmission grating is shown in Fig.1. The grating area is formed from identical thin Si plates whose pitches can be varied by changing the gaps between them. Figure 1(a) shows the whole view of the designed grating for the size of $10 \mathrm{~mm} \times 6 \mathrm{~mm}$. The grating area is $4.8 \mathrm{~mm} \times 5 \mathrm{~mm}$ and its pitch is $200 \mu \mathrm{m}$. The grating can be expanded (or shrunken) by pulling (or pushing) the handle of the grating attached with a SMA actuator. The expansion and shrinkage are generated in the plane of Fig. I(a) along the $x$ axis. Figure 1(b) shows a magnified view of the gratings in Fig.1(a). For demonstrating the feasibility of the proposed system, the pitch of the grating is designed to be relatively large ( the pitches we design are $200 \mu \mathrm{m}$ and $40 \mu \mathrm{m}$, respectively ). Moreover, to prevent the fragility of the grating, the grating line is designed to be thick by etching through the silicon wafer $(200 \mu \mathrm{m}$ in thickness). The structure having thick grating 
line will be useful to diffract light effectively in the optical configuration of Bragg diffraction when the incident light comes nearly vertically to the grating line in the plane of grating

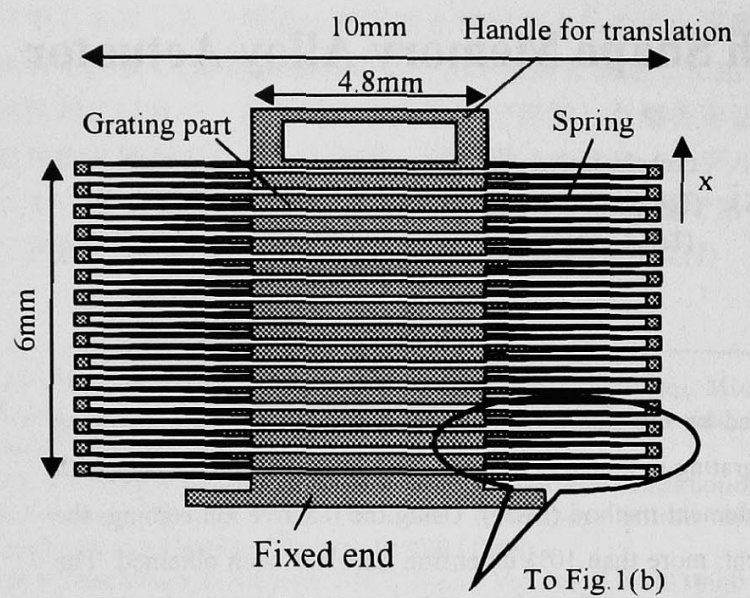

(a)

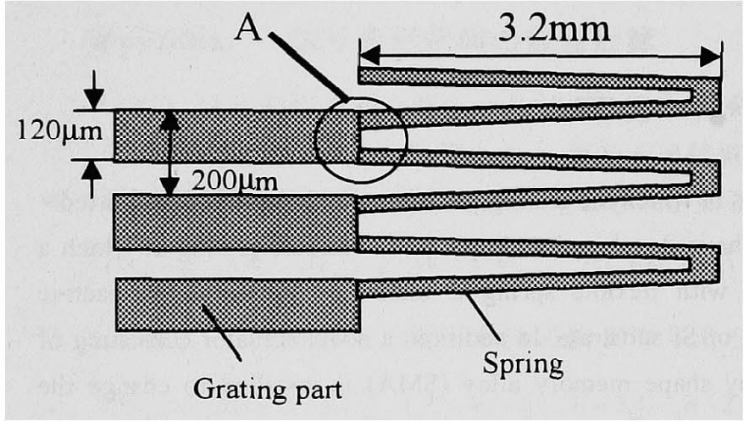

(b)

Fig. 1 Schematic diagram of the proposed pitch-variable Grating $(200 \mu \mathrm{m}$ pitch) (a) Whole view; (b) Spring part connected with grating line

The pitch-variable gratings with the spring flexures are designed to change in the pitch in a uniform way. The stress generated on the grating has been calculated by finite element method (FEM). In the baseline model for the pitch-variable grating, the chosen shape is based on moderate stress and uniform gap change. Silicon wafers are selected to fabricate the gratings due to its superior mechanical characteristics and suiting for common lithographic and processing techniques. Figure 2 shows an example of the stress distribution of the grating end simulated by FEM. It shows the stress distribution in the area indicated by A in Fig. 1 (b). The maximum stress is generated in the area where the grating plate and the springs are connected. Narrow down the width of the spring, the pitch of the grating deforms easily, but the stress in the connection area increases and the rigidity in the area A in Fig. 1(b) decreases. We decided the ratio of the width of the grating plate and that of the spring to be about 4 . Under the condition, the highest stress is $8.22 \mathrm{MPa}$ as shown in Fig.2 when the grating is expanded by $10 \%$ and the force applied to the grating is $0.98 \mathrm{~N}$. The strain value at the opposite end of the spring is almost the same as that in the area A shown in Fig.1(b).
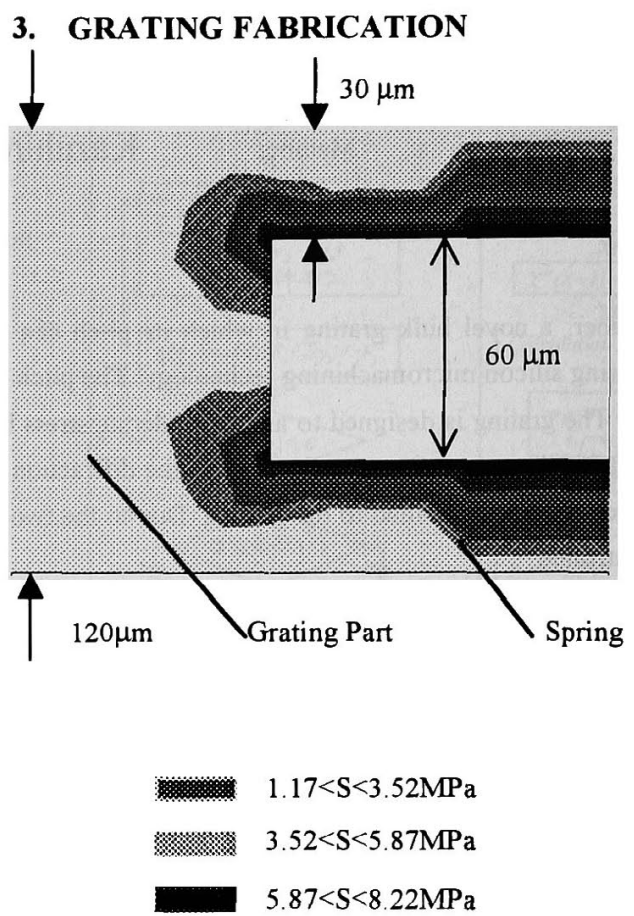

Fig.2 Stress distribution of the grating in the area $\mathrm{A}$ shown in Fig. 1 (b)

Figure 3 shows the process flow for the fabrication of the pitchvariable grating. The grating is fabricated by using one mask. The silicon substrate is $200 \mu \mathrm{m}$ thick, n-type $(100), 5-8 \Omega-\mathrm{cm}$. Firstly, the wafer is oxidized in $1100^{\circ} \mathrm{C}$ for 30 minutes. Secondly, a

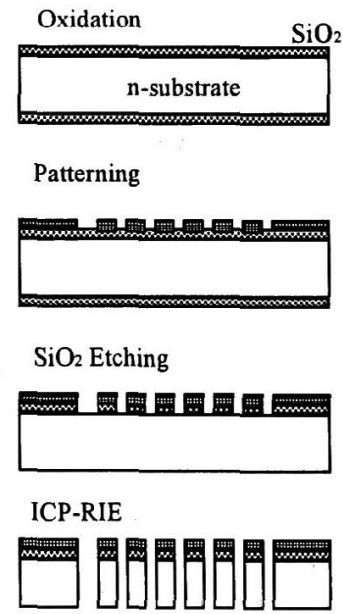

Fig. 3 The process flow of the proposed pitch-variable grating 
positive photoresist of high viscosity (OFPR-800, 450cp) is coated by spinner at the speed of $3000 \mathrm{rpm}$ to form the mask material in about $2.5 \mu \mathrm{m}$ thick. And then, lithography is carried out with usual UV-light exposure equipment ( Karl Suss, MA-8) and the exposed resist is developed in NMD-3 solution, realizing the pattern of the grating and oxidized film is etched by using HF solution to yield the mask of reactive ion etching. Lastly, the sample is etched throughout the substrate by deep reactive ion etching equipment. The process gas is a gas mixture of $\mathrm{SF}_{6}$ and $\mathrm{C}_{4} \mathrm{~F}_{8}$.

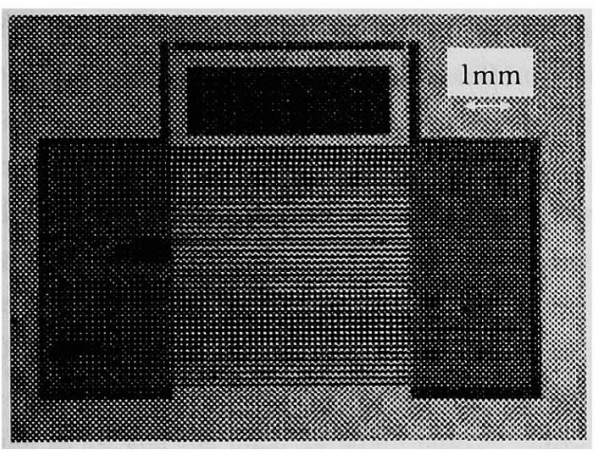

Fig.4 Fabricated pitch-variable grating

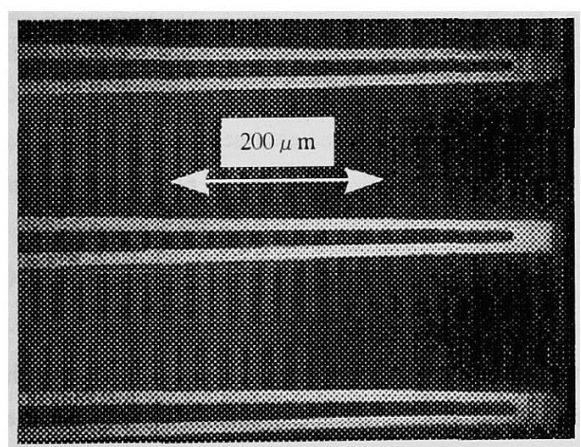

Fig.5 (a) Magnified image of the grating (spring part)

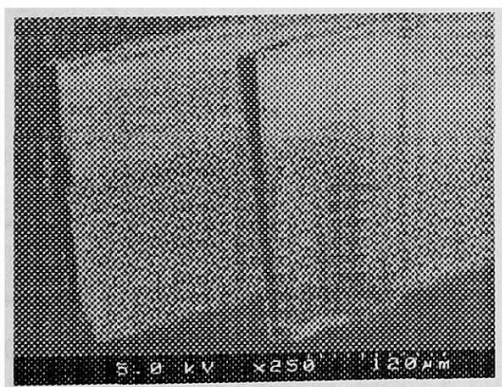

Fig.5 (b) SEM photography of the grating (spring part)

The sizes of the gratings that we have fabricated are $10 \mathrm{mmX} 6 \mathrm{~mm}$ and $2.5 \mathrm{mmX} 1.25 \mathrm{~mm}$ for the pitches of $200 \mu \mathrm{m}$ and
$40 \mu \mathrm{m}$, respectively. The photograph of the fabricated pitchvariable grating (pitch $40 \mu \mathrm{m}$ )is illustrated in Fig.4. The active grating area is in the center section with supporting spring flexures at each side. In Figs. 5 (a) and 5 (b), the magnified views of the grating spring flexures $(200 \mu \mathrm{m}$ pitch) that join the grating plates at the sides of the active grating area is shown. As shown in Fig. 5 (b), a high aspect ratio (about 10) is obtained.

\section{SHAPE MEMORY ALLOY (SMA) ACTUATOR}

A SMA actuator has now been fabricated to drive the deformable grating due to its large force output and large motion ranges. The material is consisted of $\mathrm{Ni}-\mathrm{Ti}$ alloy (approximately $50.5 \% \mathrm{Ni}$, $49.1 \% \mathrm{Ti}, 0.4 \% \mathrm{Fe}$ ). Figure 6 shows the result of the differential scanning calorimetry (DSC) of thermally treated Ni-Ti foil. It

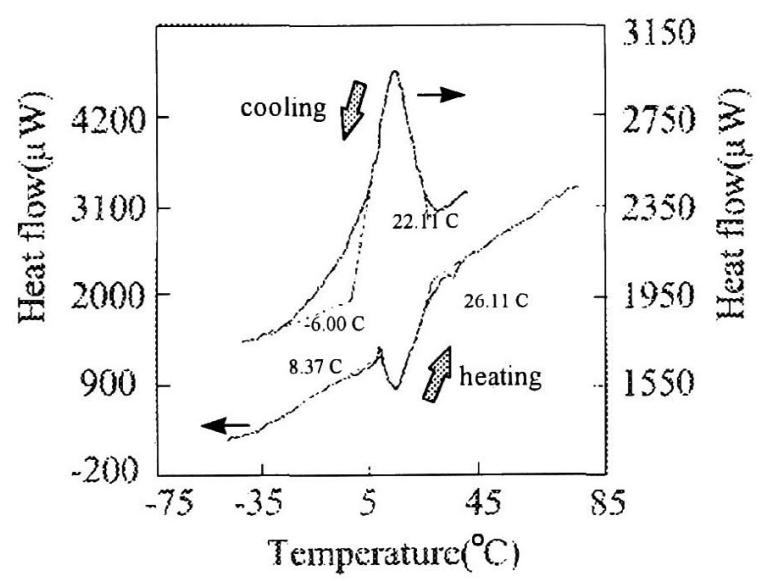

Fig.6 The result of Differential Scanning Calorimetry ( DCS )
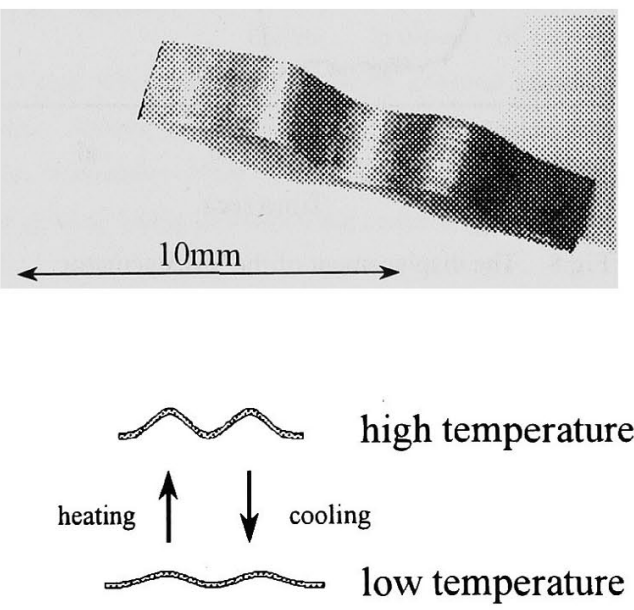

Fig. 7 SMA actuator

also shows an endothermic peak upon heating and an exothermic peak upon cooling, respectively. The result clearly shows that the thermally treated $\mathrm{Ni}$ - $\mathrm{Ti}$ foil has a shape memory 
effect. The SMA actuator is rolled to form Ni-Ti alloy foil of only $29 \mu \mathrm{m}$ in thickness as shown in Fig.7. Such a actuator is about 15 $\mathrm{mm}$ long, we have memorized the wave shape. This actuator shrinks in the high temperature phase and extends in the low temperature phase, respectively. And its maximum displacement is about $1 \mathrm{~mm}$. In order to generate the two way shape memory effect, it is aged to remove strain in $800^{\circ} \mathrm{C}$ (austenite phase) for 60 minutes and to memorize the shape in $500^{\circ} \mathrm{C}$ (martensite phase ) for 60 minutes

During the heat treatment process, the sample foil is kept in low vacuum to prevent it from oxidation. Since the SMA is able to memorize two shapes by the two-way shape memory effects, the restoring spring is unnecessary. The actuator contracts when it is heated while it extends when cooling. When proper electrical power is applied to the actuator foil, it converts the input current into thermal energy to heat the Ni-Ti foil, and the actuator converts the thermal energy into motion energy to drive the grating. Figure 8 illustrates the performance of the actuator when $0.3 \mathrm{~A}$ current as a thermal sources is applied to the alloy foil. In this case, it also shows the motion displacement is about $500 \mu \mathrm{m}$ and the response time is about 3 seconds. When the same SMA actuator is connected with the grating, it almost demonstrates the same result, so the load is not of concern for translating the grating.

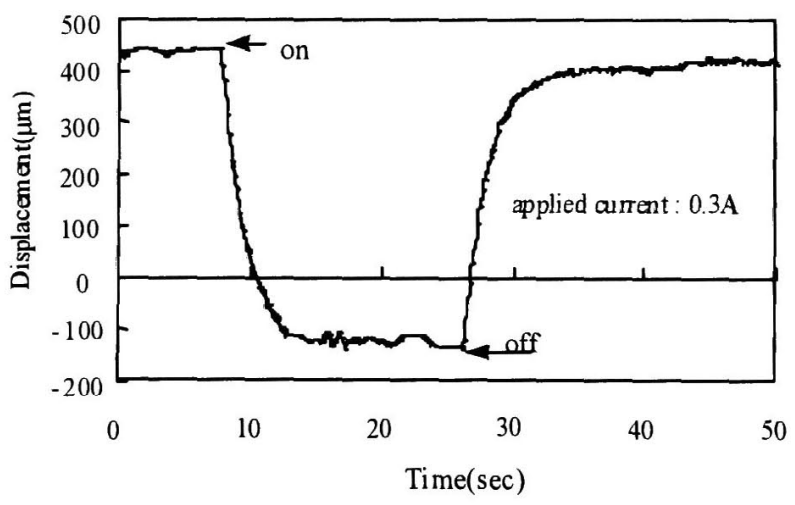

Fig 8 The displacement of the SMA actuator as a function of time

In the experiment, the SMA actuator is fixed to the handle of the fabricated grating with a hook at one end and the other end is attached to the stator. The hook can be moved along the linear guide so we can adjust the grating expansion by operating the SMA actuator. The feedback controller based on proportional and integral (PI) control circuit is designed to make the photodiode signal difference zero. To show the principle of feedback control, a photodiode position sensor with quadrant cells is used. When the laser beam is apart from the center of the sensor, the relative position of the laser spot from the center can be detected as a photocurrent. Using the photocurrent as the input signal, the SMA actuator moves by supplying the heating current until the laser beam can be aligned to the center. Figure 9 is a plot of the typical response of the SMA actuator when the feedback control is applied. There is no significant changes in the characteristics of the stability even though a long thermal cycles. The position accuracy of the SMA is about $0.5 \mu \mathrm{m}$.

\section{DIFFRACTION EXPERIMENT}

The maximum change ratio of the grating pitch has been measured by micrometer fixed to the measurement system which can be pulled or pushed manually. It can be pressed up to $1 \mathrm{~mm}$ and has been broken when the grating has been pulled to $2 \mathrm{~mm}$ in the case of $5 \mathrm{~mm}$ active long grating $(200 \mu \mathrm{m}$ pitch). The SMA actuator has been investigated to assure the alignment precision. The alignment precision is in the order of $100 \mathrm{~nm}$ when the feedback control has been utilized, which is high enough for the

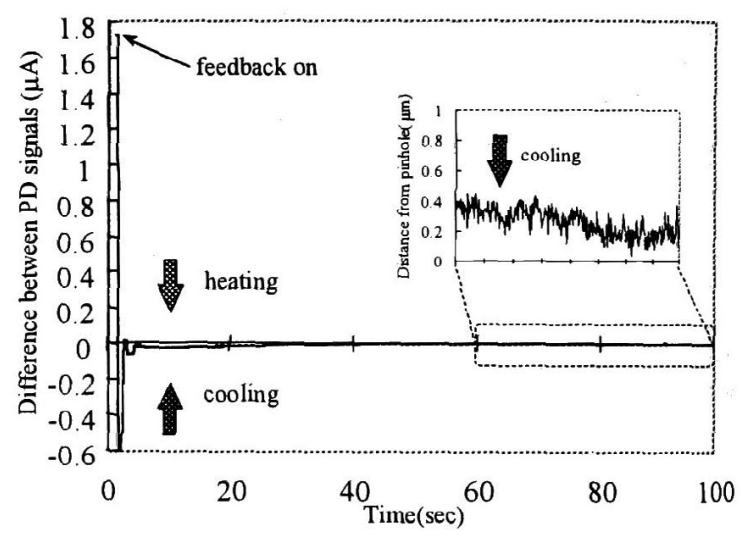

Fig.9 The stability of SMA actuator

opto-mechanical application [8]. The experimental setup is used to measure the relationship between displacement of the variable

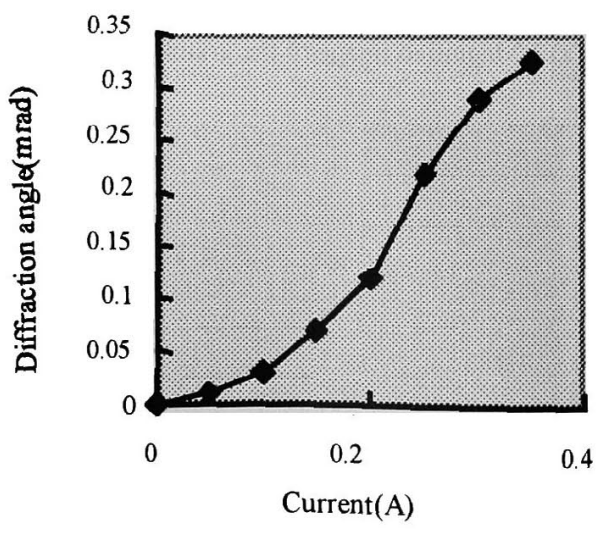

Fig.10 The diffraction angle as a function of the current applied to the SMA actuator

grating (assuming that normal condition is 0 ) and the direct 
current applied to the SMA actuator. The wave-shape actuator moves the grating when it is in the high or low temperature phase according to the supply power switch condition (on or off) along the linear guide to assure one-dimensional motion. A He-Ne laser at $632.8 \mathrm{~nm}$ wavelength illuminated the gratings. The position sensitive detector (PSD) is used as a displacement sensor to measure the position motion of the first-order light of diffraction pattern. All measurements were taken at room temperature in open air. In Figure 10, the diffraction angle of the first order light is shown as a function of the current applied to the actuator in the case of $200 \mu \mathrm{m}$ pitch grating. It shows that the grating pitch is successfully expanded by about $10 \%$ with the SMA actuator at the current of $0.35 \mathrm{~A}$.

\section{CONCLUSIONS}

The pitch-variable diffraction grating has been fabricated by reactive ion etching. The pitch-variable diffraction grating has been specially designed by FEM to assure the gap change of the grating under moderate stress. The aspect ratio of the grating plate is as high as 10 and the pitch variation ratio is more than $10 \%$. Furthermore, the grating is driven by SMA actuator to confirm the variability of diffraction angle. The delay of the response of SMA actuator is about 3 seconds when $0.3 \mathrm{~A}$ direct current is supplied. The actuator with wave shape of $15 \mathrm{~mm}$ in length and $2 \mathrm{~mm}$ in width results a $500 \mu \mathrm{m}$ peak to peak output displacement. In the diffraction experiment, more than $10 \%$ extension ratio has been obtained, which will generate the change of the diffraction angle of $0.32 \mathrm{mrad}$ in the case of $200 \mu \mathrm{m}$ pitch grating.

\section{ACKNOELEDGMENTS}

This project was supported in part by the Communication and Broadcast Program of Japan. The authors would like to acknowledge the collaboration of Mr.Yuji Arai of Tohoku University. A part of this work was performed in Venture Business Laboratory in Tohoku University.

(Manuscript received Dec.20,1999, revised May 8, 2000)

\section{REFERENCES}

[1] T.R.Ohnsteion, J.D.Zook, H.B.French, H.Guckel, T.Earles, J.Klein, P.Mangat, "Tunable IR filters with integral electromagnetic actuators," Solid-State Sensor and Actuator Workshop, Hilton Head, South Carolina, June 2-6, (1996).

[2] A.Iocco, H.G.Limberger, R.P.Salathe, "Bragg grating fast tunable filter", Elec.Lett., vol33, pp2147-2148 (1997).

[3] Min-Cheol Oh, Hyung-Jong Lee, Myung-Hyun Lee, Joo-Heon Ahn, Seon Gyu Han, and Hae-Guen Kim, "Tunable wavelength filters with Bragg gratings in polymer waveguides". Appl.
Phys.Lett.,vol.73,18, pp.2543-2545, (1998).

[4] H.Guckel, "Micro electro-mechanical positioning systems for optical instruments and switches", MOEMS97, Technical Digist,pp229-232, (1997)

[5] R.Germann, R.Beyeler, G.L.Bona, F.Horst, B.J.Offrein, H.W.M.Salemink, “ Wavelength-tunable add/drop filter for optical networks", OFC99 Technical Digest, pp268-270, (1999).

[6] D.E.Sence, J.W.Grantham, V.M.Bright, and T.H.Comtois: "Development and characterization of micro-mechanical gratings for optical modulation ," IEEE MEMS Workshop, pp.222227,(1996).

[7] D.M.Burns and V.M.Bright: " Micro-electro-mechanical variable blaze gratings", IEEE MEMS Workshop, pp.55-60, (1997).

[8] M.Sasaki, Y.Arai, and K.Hane, "Si micromachined pinhole and surrounding photodiode for Incident beam position sensing and automatic alignment", Opt. Rev. vol.5, No.2,pp115-118, (1998).

Yigui Li (Non-member) received the B.S. and M.S. degrees

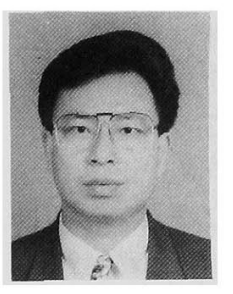
from the department of precision instrument, Harbin Institute of Technology, China in 1985 and 1991, respectively. Since 1991, he has be been a research associate of the Department of Optical-Electronic Engineering, Harbin Institute of

Technology, China. He is a currently doctoral course student of Graduate School of Mechatronics and Precision Engineering, Tohoku University. Now he is engaged in the development of optical grating using micromaching technology.

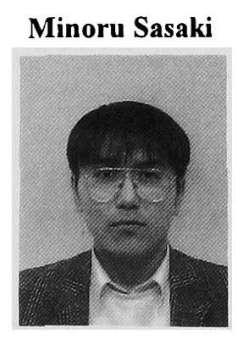

(Member) received the M.S. and Dr. Eng. Degrees from Nagoya University in 1993 and 1995, respectively. In 1996, he is a Research Fellow of the Japan Society for the Promotion of Science. Since 1996, he has been a research associate of the Department of Mechatronics and Precision Engineering, Tohoku University, and is currently engaged in the research

and development of semiconductor microsensors. $\mathrm{He}$ received the Numata Memorial Award of Japan Society for Precision Engineering in 1994. 


\section{Kazuhiro Hane (Member) received}

the M.S. and Dr. Eng. Degrees from

Nagoya University in 1980 and 1983,

Nagoya University. From 1985 to 1986 ,

he was a guest researcher of National

Research Council of Canada.

Since 1994, he has been a professor of the Department of Mechatronics and Precision Engineering, Tohoku University, and is currently engaged in the research and development of microsensors and opto-mechnical system. He received the Numata Memorial Award and the Development Award of Japan Society for Precision Engineering in 1994 and 1996, respectively 